\title{
Efficient Quantum Algorithms for State Measurement and Linear Algebra Applications
}

\author{
Apoorva Patel* and Anjani Priyadarsini ${ }^{\dagger}$ \\ Centre for High Energy Physics, Indian Institute of Science, \\ Bangalore 560012, Karnataka, India \\ *adpatel@iisc.ac.in \\ †anjanipriyav@iisc.ac.in
}

\begin{abstract}
We present an algorithm for measurement of $k$-local operators in a quantum state, which scales logarithmically both in the system size and the output accuracy. The key ingredients of the algorithm are a digital representation of the quantum state, and a decomposition of the measurement operator in a basis of operators with known discrete spectra. We then show how this algorithm can be combined with (a) Hamiltonian evolution to make quantum simulations efficient, (b) the Newton-Raphson method based solution of matrix inverse to efficiently solve linear simultaneous equations, and (c) Chebyshev expansion of matrix exponentials to efficiently evaluate thermal expectation values. The general strategy may be useful in solving many other linear algebra problems efficiently.
\end{abstract}

Keywords: Chebyshev polynomials; computational complexity; digital representation; Newton-Raphson method; quantum simulations.

\section{Introduction}

Simulations of quantum systems on classical computers are hard, but they are expected to become easy on quantum computers by converting their parallel implementations to superpositions - that was pointed out by Feynman as a major motivation for developing quantum computers [1. Such simulations would model physical systems directly into the quantum hardware, but with greater freedom in the choice of parameters than the limited values the natural systems have. That can be of great help in understanding their dynamical properties, and this is a likely area where quantum computers will demonstrate their superiority over classical ones in near future.

Simulation problems are function evaluation problems. Their computational complexity has to be measured in terms of both the input size and the output precision. Efficient algorithms are those with the computational complexity polynomial in the input as well as the output number of bits. Conventional computational complexity analysis focuses on input size dependence of decision problems (with just one output bit), and study of output efficient algorithms is relegated to design of optimal methods for arbitrary precision numerical analysis 2. Optimisation of output precision dependence of quantum simulation problems has attracted attention relatively recently [3, 4, 5]. In this context, we have defined the computational complexity class P:P, which is the set of computational problems that can be solved with resources polynomial in the input size as well as the output size [5]. In the present work, we demonstrate that several practical quantum linear algebra problems belong to this class.

Many simulation problems can be expressed as time evolution under specified interactions, from some simple initial state to the final state whose properties are to be determined. In this setting, the Hamiltonian evolution problem has been extensively investigated. Consider a many-body quantum system. Quantum simulations can sum multiple evolutionary paths contributing to a quantum process in superposition at one go, while classical simulations need to evaluate these paths one by one. Real physical systems are often governed by local Hamiltonians, i.e. where each component interacts with only a limited number of its neighbours, independent of the overall size of the system. Early quantum evolution algorithms exploited this property for efficient use of time and space resources 6, 7. More recently, the error complexity of the evolution has been reduced from power-law to logarithmic in the inverse error, using algorithms with large time evolution steps [3, 4, 5]. 
Classical simulation algorithms find it convenient to separate the evolution and the measurement parts. That is logical, since all properties of the final state are accessible in classical computation. But the situation is different for quantum simulations; the solution of any quantum simulation problem is incomplete without a procedure to measure the desired final state observables. Explicitly, in the $2^{n}$-dimensional Hilbert space of $n$ qubits, we can superpose $2^{n}$ components evolving in parallel, but we can measure only $n$ binary observables at the end. So the exponential gain of superposition is limited by the restriction to extract only a small number of results at the end. This dichotomy means that quantum algorithms will be advantageous only when the final observables are local in some manner, and no general prescription is available.

The problem of how to efficiently measure expectation values of final state observables, after the time evolution, has not been adequately investigated in the existing literature. Since quantum measurements are inherently probabilistic, determination of the expectation values needs multiple repetitions of the same algorithm. Thereafter, importance sampling or phase estimation based methods yield errors that decrease as power-laws in the number of repetitions [8, 9], and that is not efficient. What we want is a strategy that decreases the errors exponentially with the number of repetitions. While that is not possible for generic observables, it can be achieved for $k$-local observables that appear in evaluations of $k$-point Green's functions for many-body systems. In what follows, we explicitly show how to do that.

To construct our algorithms, we first look at well-known output efficient classical algorithms, and select from them those that can be maximally parallelised. Then we convert the parallel evaluation structure to quantum superposition, to make the algorithms input efficient as well. In this strategy, the mathematical techniques used in various components of the algorithms are familiar; we just put them together in a clever way. It is worth noting that the best classical algorithms may not offer the best parallelisation that can be exploited by quantum superposition. In such situations, our best quantum algorithms are related to the maximally parallelisable classical algorithms, and are unrelated to the best classical algorithms.

We first explain our digital quantum computation framework in Section 2, expanding on our previous presentation [5]. Then, we describe our efficient measurement procedure in Section 3, in the context of quantum simulations. Afterwards, in Sections 4 and 5 respectively, we combine it with an algorithm for matrix inverse to solve simultaneous linear equations, and an algorithm for matrix exponentiation to evaluate thermal expectation values. In all cases, we compare the computational complexity of our results with known classical and quantum algorithms, and point out the improvements made. Our results for the computational complexity of measuring $k$-point Green's functions, of evolving a quantum state with the given Hamiltonian, of solving linear simultaneous equations and of exponentiating a sparse matrix, are contained in Eqs.301844159) respectively.

\section{Digital Quantum Computation}

It is routine to represent a quantum state in an $N$-dimensional Hilbert space as

$$
|x\rangle=\sum_{j=0}^{N-1} x_{j}|j\rangle, \quad \sum_{j=0}^{N-1}\left|x_{j}\right|^{2}=1,
$$

where $x_{j}$ are continuous complex variables. This analog representation is not convenient for high precision calculations, because any physical apparatus can determine continuous values up to only a limited precision. A digital representation bypasses this limitation, by breaking up $x_{j}$ into a sequence of digits, where each digit has only a finite number of possibilities that can be easily distinguished, and the number of digits can be made as large as desired. Of course, the benefit of digitisation is maximised when the complete calculation, from the input to the output, is carried out in the digital representation. We describe here the ingredients needed to execute a quantum computation in such a digital mode.

States: We use the digital representation specified by the map [5]:

$$
|x\rangle \rightarrow \frac{1}{\sqrt{N}} \sum_{j=0}^{N-1}|j\rangle\left|x_{j}\right\rangle_{q},
$$

which mimics the storage of a vector in classical computer registers. It is a quantum state in a $\left(2^{q} N\right)$ dimensional Hilbert space, where $\left|x_{j}\right\rangle_{q}$ are the basis vectors of a $q$-bit register representing the truncated values of $x_{j}$ (a complex number $x_{j}$ can be represented by a pair of real numbers, and all $2^{q} x_{j}$ are truncated to integers). This representation is fully entangled between the component index state $|j\rangle$ and the register 
value state $\left|x_{j}\right\rangle_{q}$, with a unique non-vanishing $\left|x_{j}\right\rangle_{q}$ (out of $2^{q}$ possibilities) for every $|j\rangle$. It is important to observe that no constraint is necessary on the register values $x_{j}$ in this representation- the algorithm has to take care of the overall unitary evolution. This freedom allows simple implementation of all linear algebra operations (and not just unitary transformations) on $\left|x_{j}\right\rangle_{q}$, transforming them among the $2^{q}$ basis states using only C-not and Toffoli gates of classical reversible logic, with the index state $|j\rangle$ acting as control. For example,

$$
\begin{aligned}
c|x\rangle & \rightarrow \frac{1}{\sqrt{N}} \sum_{j=0}^{N-1}|j\rangle\left|c x_{j}\right\rangle_{q}, \\
|x\rangle+|y\rangle & \rightarrow \frac{1}{\sqrt{N}} \sum_{j=0}^{N-1}|j\rangle\left|x_{j}+y_{j}\right\rangle_{q},
\end{aligned}
$$

map non-unitary operations on the left to unitary operations on the right. These elementary operations can be combined to construct any power series 11 Note that a crucial requirement for implementing linear algebra operations in the digital representation is that only a single index (" $j$ " in the preceding formulae) controls the whole entangled state.

Observables: The freedom to choose a convenient representation for the quantum states is particularly useful due to the fact that the quantum states are never physically observed. All physically observed quantities are the expectation values of the form $\left\langle x\left|O_{a}\right| x\right\rangle$. So the digital representation is completed by constructing for each observable $O_{a}$ in the $N$-dimensional Hilbert space a related observable $\tilde{O}_{a}$ in the $\left(2^{q} N\right)$-dimensional Hilbert space, such that

$$
\left\langle x\left|O_{a}\right| x\right\rangle=\sum_{j, l=0}^{N-1} x_{j}^{*} x_{l}\left\langle j\left|O_{a}\right| l\right\rangle=\frac{1}{N} \sum_{j, l=0}^{N-1}{ }_{q}\left\langle x_{j}\left|\left\langle j\left|\tilde{O}_{a}\right| l\right\rangle\right| x_{l}\right\rangle_{q} .
$$

For this equality to hold, it suffices to construct the operator $\tilde{O}_{a}=O_{a} \otimes O_{q}$, where the Hermitian operator $O_{q}$ in the $2^{q}$-dimensional Hilbert space satisfies

$$
\left\langle x_{j}\left|O_{q}\right| x_{l}\right\rangle=N x_{j}^{*} x_{l}
$$

To this end, we note that

$$
\left\langle x_{j}\left|\left(1+\sigma_{1}\right)^{\otimes q}\right| x_{l}\right\rangle=1,
$$

and the place-value operator for a bit-string,

$$
V=\sum_{k=0}^{q-1} 2^{-k} I^{\otimes k} \otimes\left(\frac{1-\sigma_{3}}{2}\right) \otimes I^{\otimes(q-k-1)},
$$

gives $V\left|x_{j}\right\rangle=x_{j}\left|x_{j}\right\rangle$. The solution to Eq.(6), therefore, is independent of the quantum state and the observable:2

$$
O_{q}=N V^{\dagger}\left(1+\sigma_{1}\right)^{\otimes q} V
$$

Since $V$ is a sum of $q$ bit-wise fully factorised terms, $O_{q}$ can be expressed as a sum of $q^{2}$ such terms, and the computational complexity of measurement of physical observables in the digital representation is $O\left(q^{2}\right)$ times that in the analog representation. Moreover, the bit-wise separated structure of $V$ allows evaluation of any single specific bit of $\left\langle x\left|O_{a}\right| x\right\rangle$, if so desired, with $O(q)$ extra effort compared to the analog case. More generally, any function $f\left(x_{j}\right)$ for the state $\left|x_{j}\right\rangle$ can be computed using just the machinery of classical reversible logic, and overall normalisations can be adjusted at the end of the calculation.

We essentially bypass the constraint of unitarity in the digital representation, by using two different metrics in the $2^{q}$-dimensional space of the coordinates $\left\{x_{j}\right\}$. The Cartesian metric is used for implementing the linear algebra operations, and the metric $O_{q}$ is used for evaluating the expectation values of observables. This trick allows us to exploit the advantages of the digital representation over the analog one, i.e. easy implementation of arbitrary precision calculations and simple linear algebra operations. It is worthwhile to

\footnotetext{
${ }^{1}$ This is a much simpler procedure than the "addition of unitaries" used in Ref. [4].

${ }^{2}$ In terms of the uniform superposition state $|s\rangle_{q}=H^{\otimes q}|0\rangle_{q},\left(1+\sigma_{1}\right)^{\otimes q}=2^{q}|s\rangle_{q}\langle s|$. Also, the factor $\left(1+\sigma_{1}\right)^{\otimes q}$ can be omitted from $O_{q}$ in evaluation of $\left\langle x_{j}\left|O_{q}\right| x_{j}\right\rangle$.
} 
note that construction of fault-tolerant operations in the digital representation is considerably simpler than in the analog case, because only a small set of quantum logic gates is required - the C-not and the Toffoli gates to implement Eqs.(344) and the Hadamard gate to implement Eq.(10).

Initialisation: To efficiently incorporate the digital representation in an algorithm, methods must be found to not only manipulate the register values $\left|x_{j}\right\rangle$ efficiently, but also to initialise and to observe them. At the start of the calculation, we need to assume that the initial values $x_{j}(0)$ can be efficiently computed from $j$, say using the control operation $C_{x}$. Then, for $N=2^{n}$, the initial state is created easily using the Hadamard and the $C_{x}$ operations as

$$
\begin{aligned}
|0\rangle|0\rangle_{q} \quad & \stackrel{H^{\otimes n} \otimes I}{\longrightarrow} \frac{1}{\sqrt{N}} \sum_{j=0}^{N-1}|j\rangle|0\rangle_{q} \\
& \stackrel{C_{x}}{\longrightarrow} \frac{1}{\sqrt{N}} \sum_{j=0}^{N-1}|j\rangle\left|x_{j}(0)\right\rangle_{q} .
\end{aligned}
$$

When $N$ is not a power of 2 , a simple fix is to enlarge the $j$-register to the closest power of 2 and initialise the additional $x_{j}$ to zero. Thereafter, the linear algebra operations can be implemented such that the additional $x_{j}$ remain zero, and the overall normalisation (i.e. $1 / \sqrt{N}$ ) can be corrected in the final result as a proportionality constant.

Evolution: The evaluation of $|x(T)\rangle=U|x(0)\rangle$ for a unitary operator $U$ is a matrix-vector product. That can be efficiently calculated in the digital representation, when $U$ can be expressed as a sum (or product) of a finite number of block-diagonal terms, e.g. using a series expansion. The linear algebra operations can then combine all the terms easily. It is not necessary for the intermediate steps involving individual terms to satisfy the unitary constraint; it is sufficient that the final result obeys $\sum_{j=0}^{N-1}\left|x_{j}(T)\right|^{2}=1$.

Measurement: At the end of the calculation, we need to assume that the final state observables are efficiently computable from $x_{j}(T)$. In the analog representation, determination of $\left|x_{j}(T)\right|^{2}$ is probabilistic, and requires an ensemble of measurements covering the full range of the index $j$. In contrast, in the digital representation, when a particular $j$ is observed, the corresponding $x_{j}(T)$ can be determined exactly. Then, the advantage of the digital representation is that the index $j$ can be handled in parallel (classically) or in superposition (quantum mechanically). The fact that $V$ is an eigenoperator for $\left|x_{j}\right\rangle$ allows $\left\langle x_{j}\left|O_{q}\right| x_{l}\right\rangle$ to be evaluated deterministically with $O\left(q^{2}\right)$ effort. That makes the efficiently measurable observables those for which the sum over $N^{2}$ terms in Eq.(5) can be evaluated with poly $(n)$ effort.

Density matrix: We point out that a digital representation for the density matrix can be constructed in a completely analogous manner. The map for

$$
\rho=\sum_{i, j=0}^{N-1} \rho_{i j}|j\rangle\langle i|, \quad \sum_{i=0}^{N-1} \rho_{i i}=1
$$

describing pure as well as mixed states, is

$$
\rho \rightarrow \frac{1}{N} \sum_{i, j=0}^{N-1}|j\rangle\left\langle i|| \rho_{i j}\right\rangle_{q}
$$

where $\left|\rho_{i j}\right\rangle_{q}$ are the basis vectors of a $2^{q}$-dimensional Hilbert space representing the truncated values of $\rho_{i j}$. The most general evolution of the density matrix is a completely positive trace-preserving linear transformation, specified by a Kraus representation

$$
\rho \rightarrow \sum_{\mu} M_{\mu} \rho M_{\mu}^{\dagger}, \quad \sum_{\mu} M_{\mu}^{\dagger} M_{\mu}=1
$$

It is straightforward to implement that with operations similar to Eqs. (334). Furthermore, expectation value of any physical observable can be obtained as

$$
\operatorname{Tr}\left(\rho O_{a}\right)=\sum_{i, j=0}^{N-1} \rho_{i j}\left\langle i\left|O_{a}\right| j\right\rangle=2^{q / 2} \sum_{i, j=0}^{N-1}\left\langle i\left|O_{a}\right| j\right\rangle_{q}\left\langle s|V| \rho_{i j}\right\rangle_{q} .
$$


Thus any single specific bit of $\operatorname{Tr}\left(\rho O_{a}\right)$ can be evaluated with the same effort as in the analog case, and the computational complexity of measurement of an observable in the digital representation is $O(q)$ times that in the analog representation.

Computational complexity: Finally, we have to take care of the fact that a digital computation with finite register size produces round-off errors, because real values are replaced by integer approximations. With $q$-bit registers, the available precision is $\delta=2^{-q}$. Using simple-minded counting, elementary bit-level computational resources required for additions, multiplications and polynomial evaluations are $O(q), O\left(q^{2}\right)$ and $O\left(q^{3}\right)$ respectively. (Overflow/underflow limit the degree of the polynomial to be at most $q$.) Since all efficiently computable functions can be approximated by accurate polynomials, the effort needed to evaluate individual elements of any operator is thus $O\left(q^{3}\right)$.

Linear algebra algorithms are often dominated by operator-state products. For $d$-sparse operators, their classical computational complexity is $O\left(d N q^{3}\right)$. Such operators can be expressed as a sum of $d$ block-diagonal operators with fixed block sizes, and the number of blocks is $O(N)$. When an efficient labeling scheme for the blocks exists, the index $j$ can be broken down into $O(n)$ tensor product factors (analogous to Eq. (10)), and then quantum superposition makes the cost of multiplying the operator with a state proportional to $n$. That makes the quantum computational complexity of the operator-state product $O\left(d n q^{3}\right)$.

The register size $q$ is determined using the constraint that the round-off error accumulated over the whole algorithm should not exceed the specified error bound $\epsilon$. For an algorithm containing $r$ sparse operator-state products, that can be achieved by choosing $d r \delta=O(\epsilon)$, which gives $q=\Omega(\log (d r / \epsilon))$. This relation between $q$ and $\epsilon$ is crucial in construction of algorithms that are efficient with respect to the output precision; a computational complexity of the form poly $(q)$ becomes poly $(\log \epsilon)$.

\section{Efficient Measurements}

Hamiltonian simulation evolves an initial quantum state $|\psi(0)\rangle$ to a final quantum state $|\psi(T)\rangle$, in presence of interactions specified by a Hamiltonian $H(t)$ :

$$
|\psi(T)\rangle=U(T)|\psi(0)\rangle, \quad U(T)=\mathcal{P}\left[\exp \left(-i \int_{0}^{T} H(t) d t\right)\right] .
$$

The initial state is usually easy to prepare, while the final state is generally unknown. The path ordering of the unitary evolution operator $U(T)$, denoted by the symbol $\mathcal{P}$ in Eq.(16), is needed when various terms in the Hamiltonian do not commute. Properties of the final state are subsequently extracted from expectation values of observables:

$$
\langle O\rangle=\langle\psi(T)|O| \psi(T)\rangle .
$$

Efficient algorithms to determine the final state $\psi(T)$, up to a specified error bound $\epsilon$ and for a certain class of Hamiltonians, have been constructed in earlier works [3, 4, 5]. They have computational complexity

$$
O\left(T \frac{\log (T / \epsilon)}{\log (\log (T / \epsilon))} \mathcal{C}\right)
$$

where $\mathcal{C}=O\left(d n q^{3}\right)$ is the computational complexity of evaluating a sparse Hamiltonian-state product. Here, assuming that $\psi(T)$ is available, we formulate a method to determine the expectation values $\langle O\rangle$ efficiently, up to a given precision $\epsilon$ and for a certain class of observables. Together, they make a variety of quantum simulation problems belong to the computational complexity class P:P.

We concern ourselves here only with bounded operators 3 acting in finite $N$-dimensional Hilbert spaces. A general operator would then be a dense $N \times N$ matrix, and there is no efficient way to even write it down. So we only look at operators with the following properties, often encountered in physical problems:

(1) The Hilbert space is a tensor product of many small components of fixed size, e.g. $N=2^{n}$ for a system of $n$ qubits. Generically, $|\psi(T)\rangle$ is an entangled state in this space.

(2) The operator is a tensor product of a finite number of local variables, e.g. $O=\prod_{i=1}^{k} O_{i}$ where each $O_{i}$ is a single qubit observable at a distinct location. More generally, each $O_{i}$ can be spread over a fixed number of neighbouring components. Such operators appear in evaluations of $k$-point Green's functions in many-body systems, and we call them $k$-local.

(3) The decomposition of each $O_{i}$ in terms of its elementary components is efficiently computable, e.g. $O_{i}$

\footnotetext{
${ }^{3}$ Physical problems with unbounded operators exist, but their numerical solutions require more sophisticated techniques.
} 
for a qubit is a linear combination of Pauli operators with specific coefficients (which may depend on $i$ ). These features allow a compact description of the observable $O$, and then the resources required to just write it down do not influence determination of its expectation value. Furthermore, evaluation of the sparse matrix-vector product $O|\psi(T)\rangle$ can be easily parallelised, if necessary.

In terms of these specifications, operator expectation values for problems in class P:P can be calculated using computational resources that are polynomial in $\log (N)$ and $\log (\epsilon)$, with finite $k$.

\subsection{Operator Decomposition}

Our efficient measurement strategy has two important ingredients. The first ingredient is to decompose the operator as a sum of tensor products of Pauli operators. Any single qubit $O_{i}$ can be expressed in the Pauli basis as $\left(a_{0} I+a_{1} \sigma_{x}+a_{2} \sigma_{y}+a_{3} \sigma_{z}\right)_{i}$. In this basis, we then have the decomposition:

$$
O=\prod_{i=1}^{k} O_{i}=\sum_{j=1}^{K} \beta_{j} \Sigma_{j},
$$

where each $\Sigma_{j}$ is a tensor product of $k$ Pauli operators at different locations, with $K \leq 3^{k}$. Also, a Hermitian $O$ implies that $\beta_{j}$ are real. When $O_{i}$ is spread over a finite cluster of $s$ qubits, its Pauli basis decomposition has $4^{s}$ terms, and the corresponding Eq.(19) has at most $K=4^{k s}$ terms. The important point is that $K$ is finite when $k$ is finite.

In a more rigorous notation, one has to write a tensor product factor $I$ at each of the $n-k$ locations not covered by $O_{i}$. When calculating the expectation values, these $n-k$ locations get summed over, resulting in a reduced density matrix for the $k$ locations of $O_{i}$, and the non-trivial part of measurement depends on this reduced density matrix only. For the sake of simplicity, we avoid such an elaborate notation.

With our assumed properties of $O, K$ is a finite number and the coefficients $\beta_{j}$ are easily computable (henceforth we take them as known). The advantage of this decomposition are:

(a) All $\Sigma_{j}$ have well-separated eigenvalues \pm 1 only, and their eigenvectors are known. That simplifies their measurement [10].

(b) The tensor product factors are decoupled, and so can be evaluated in parallel. The value of $\Sigma_{j}$ can be accumulated in a single ancilla qubit with $O(k)$ operations, which can then be determined by a single binary measurement operation 4 This is completely analogous to the syndrome extraction procedure for quantum error correction codes 11 .

The linear norm of $O$ is, $\|O\|=\sum_{j}\left|\beta_{j}\right| \cdot\left\|\Sigma_{j}\right\|=\sum_{j}\left|\beta_{j}\right|$. Various $\Sigma_{j}$ may not commute with each other, and so the $\left\langle\Sigma_{j}\right\rangle$ need to be evaluated one by one. When each $\left\langle\Sigma_{j}\right\rangle$ is determined up to an additive error $\epsilon$, the expectation value $\langle O\rangle$ is determined up to an accuracy

$$
\epsilon\langle O\rangle \leq \sum_{j}\left|\beta_{j}\right| \epsilon=\epsilon\|O\|
$$

With these definitions, the total computational complexity for determination of $\langle O\rangle$ to fractional error $\epsilon$ is $O(K \mathrm{~km})$, where $m$ is the number of measurement trials needed to determine each $\Sigma_{j}$ to additive error $\epsilon$.

\subsection{Measurement Optimisation}

Consider evaluation of a single $\left\langle\Sigma_{j}\right\rangle$ in quantum theory. Quantum measurements are probabilistic, and one has to repeat the process many times, with identically prepared states, to obtain the result with high accuracy. Individual measurements of $\Sigma_{j}$ yield binary results. Let $p, 1-p$ be the probabilities that $\Sigma_{j}$ is measured to be $+1,-1$ respectively. Then

$$
\left\langle\Sigma_{j}\right\rangle=2 p-1
$$

i.e. the expectation value of $\left(1+\Sigma_{j}\right) / 2$ is the probability that $\Sigma_{j}$ is measured to be +1 . Logic circuits for measuring $\Sigma_{j}$ are easily constructed, based on the facts that $\Sigma_{j}$ is unitary and $\left(1 \pm \Sigma_{j}\right) / 2$ are projection operators. We use the $\sigma_{z}$ eigenstates, $|0\rangle$ and $|1\rangle$, as the computational basis. Then the $\sigma_{x}, \sigma_{y}$ eigenvalues can be extracted by measurements in suitably rotated bases, using the identities $\sigma_{x}=H \sigma_{z} H, \sigma_{y}=i \sigma_{z} \sigma_{x}$, where $H$ is the Hadamard operator. It is straightforward to collect contributions of different Pauli factors

\footnotetext{
${ }^{4}$ This is a far superior strategy compared to performing $k$ binary measurements.
} 


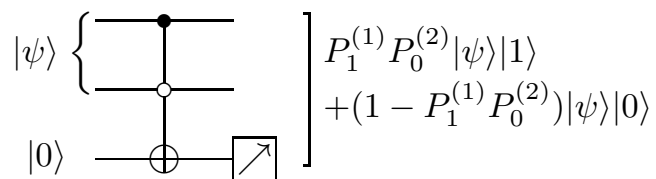

(a)

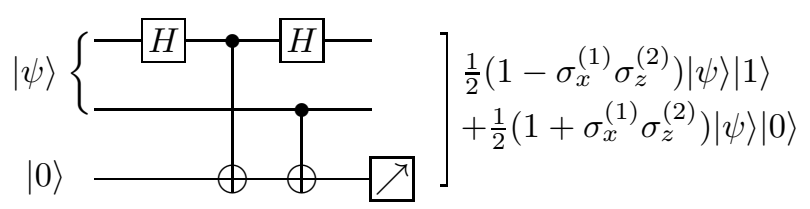

(b)

Figure 1: Quantum logic circuits for measuring (a) a product of two projection operators, and (b) a product of two Pauli operators. $\nearrow$ denotes the binary measurement operation.

making up $\Sigma_{j}$ into a single ancilla qubit using controlled operations. Finally, binary measurement of the ancilla qubit in the computational basis yields the value for $\Sigma_{j},+1$ or -1 . As a simple illustration, Figs.11a,b show the quantum logic circuits for measuring a product of two projection operators and a product of two Pauli operators respectively.

We obtain the accuracy with which $\left\langle\Sigma_{j}\right\rangle$ can be determined after $m$ binary measurements, according to the well-known Chernoff bound 12 . Let $X_{i}$ be independent random variables that take values +1 or -1 , with probability $p$ and $1-p$ respectively. Then their mean over $m$ determinations, $\bar{X}=\frac{1}{m} \sum_{i=1}^{m} X_{i}$, converges to $\mu=2 p-1$ as $m \rightarrow \infty$. With finite $m$, and $s>0$,

$$
\operatorname{Prob}(\bar{X}-\mu>\delta)=\operatorname{Prob}\left(e^{s \sum_{i} X_{i}}>e^{s m(\mu+\delta)}\right)=\left\langle e^{s \sum_{i} X_{i}}\right\rangle e^{-s m(\mu+\delta)},
$$

by Markov's inequality. Evaluation of the expectation value gives

$$
\left\langle e^{s \sum_{i} X_{i}}\right\rangle=\prod_{j=1}^{m}\left(p e^{s}+(1-p) e^{-s}\right) \leq e^{-m s} e^{m p\left(e^{2 s}-1\right)} .
$$

Optimisation of the bound in Eq.(22), with respect to the parameter $s$, gives $e^{2 s}=1+\Omega$ where $\Omega=\frac{\delta}{2 p}$. The overall bound for the upper tail probability then becomes, with $0 \leq \delta \leq 2(1-p)$,

$$
\operatorname{Prob}(\bar{X}-\mu>\delta) \leq\left(\frac{e^{\Omega}}{(1+\Omega)^{1+\Omega}}\right)^{m p} .
$$

A similar analysis for the lower tail probability gives, with $0 \leq \delta \leq 2 p$,

$$
\operatorname{Prob}(\bar{X}-\mu<-\delta) \leq\left(\frac{e^{-\Omega}}{(1-\Omega)^{1-\Omega}}\right)^{m p}
$$

Using the inequalities,

$$
\ln (1+\Omega)>\frac{\Omega}{1+\Omega / 2}, \quad(1-\Omega) \ln (1-\Omega)>-\Omega+\frac{\Omega^{2}}{2},
$$

the combined failure probability satisfies 5

$$
\operatorname{Prob}(|\bar{X}-\mu|>\delta)<2 e^{-m p \Omega^{2} /(2+\Omega)} .
$$

To make this failure probability less than a specified value $\epsilon_{m}$, we need

$$
m>\ln \left(2 / \epsilon_{m}\right)\left(\frac{2+\Omega}{p \Omega^{2}}\right)=\ln \left(2 / \epsilon_{m}\right)\left(\frac{8 p+2 \delta}{\delta^{2}}\right) .
$$

The $O(K k m)$ computational complexity for measurement then has logarithmic dependence on $\epsilon_{m}$ as desired. Note also that the total success probability for determination of $\langle O\rangle$ exceeds $\left(1-\epsilon_{m}\right)^{K}>1-K \epsilon_{m}$, which is greater than $1 / 2$ for $\epsilon_{m}<1 /(2 K)$.

There is a problem, however, when $\mu$ is a continuous variable. That would require small values of $\delta$ for high accuracy, and the bound in Eq.(28) behaves as $1 / \delta^{2}$ as $\delta \rightarrow 0$. This power law scaling is certainly undesirable. A way out is to make the possible values of $\mu$ discrete. Then $\delta$ can be given a finite value, e.g. half the separation between the discrete values of $\mu$, and one does not have to worry about how the total computational complexity depends on it. Such a discretised measurement process can be constructed using the digital representation of quantum states, and we turn to that in the next subsection.

\footnotetext{
${ }^{5}$ For binary random variables taking values 1 or 0 , the bound is the same with $\Omega=\delta / p$.
} 


\subsection{Discrete Optimal Measurements}

In our digital representation described in Section 2

$$
\left\langle x\left|\Sigma_{i}\right| x\right\rangle=\frac{1}{N} \sum_{j, l=0}^{N-1}\left\langle j\left|\Sigma_{i}\right| l\right\rangle\left\langle x_{j}\left|O_{q}\right| x_{l}\right\rangle .
$$

In this expression, the quantities $\left\langle j\left|\Sigma_{i}\right| l\right\rangle$ are fixed constants that can be evaluated at the outset, independent of the state $|x\rangle$. Given the tensor product structure of $\Sigma_{i}$, several simplifications can be carried out:

(1) The eigenbasis of $\Sigma_{i}$ is known. Performing measurements in this basis reduces the double sum on r.h.s. of Eq.(29) to a single one, i.e. $j=l$.

(2) $\left\langle j\left|\Sigma_{i}\right| j\right\rangle$ are products of $k$ nontrivial factors, and so can be easily evaluated for all values of $j$.

(3) Since $\Sigma_{i}$ have eigenvalues \pm 1 , and $\langle x \mid x\rangle=1$, it is sufficient to evaluate contribution of one of the eigenvalues to $\left\langle\Sigma_{i}\right\rangle$, which amounts to restricting the sum on the r.h.s. of Eq.(29) to a subset of terms.

When a particular value of $\Sigma_{i}$ is extracted into an ancilla qubit, say as illustrated in Fig.1(b), and a binary measurement of the ancilla qubit is performed, all the unmeasured qubits are automatically traced over. This partial trace gives the sum of the corresponding terms on the r.h.s. of Eq.(29), adding up to $p$ or $1-p$. Next, as per Eqs. (89), $O_{q}$ is a sum of $q^{2}$ projection operators with place-value weights, and each of the $q^{2}$ terms can be extracted using an ancilla qubit as illustrated in Fig.1(a). Moreover, in the digital representation, $\left|x_{j}\right\rangle$ is an eigenstate of $V$, and so the result in the ancilla qubit is deterministic (and not probabilistic). Adding all the $q^{2}$ results with their place-value weights, which can be done classically, gives the total result for $\left\langle x_{j}\left|O_{q}\right| x_{j}\right\rangle$.

Combining measurements of both $\Sigma_{i}$ and $O_{q}$, we obtain $p$ in a digital representation. Since each bit of $p$ can only take the two discrete values, 0 or 1 , it can be determined with high confidence (i.e. small $\epsilon_{m}$ ) and a coarse-grained measurement window (i.e. $\delta=1 / 2$ ), in the notation of the previous subsection. The crucial advantage provided by the digital representation is that a probabilistic estimate of $p$ is replaced by a deterministic evaluation of $p$, which can be carried out bit-by-bit to any desired accuracy.

In this evaluation strategy, we have broken up $O$ as a sum of $O\left(K q^{2}\right)$ discrete operators. Measurement of $\Sigma_{i}$ requires $O(k)$ effort, measurement of an individual term of $V$ requires $O(q)$ effort, and the evaluation of each bit of $p$ is repeated $m=O\left(\log \left(1 / \epsilon_{m}\right)\right)$ times as in the previous subsection. The total measurement effort is, therefore, $O\left(K q^{2} \cdot k q \cdot q m\right)=O\left(K k q^{4} m\right)$. To evaluate $\langle O\rangle$ with fractional error $\epsilon$, we need $q=O(\log (1 / \epsilon))$, as in Eq.(20). That makes the overall measurement complexity for evaluation of $\langle O\rangle$

$$
O\left(K k q^{4} m\right)=O\left(K k \log ^{4}(1 / \epsilon) \log \left(1 / \epsilon_{m}\right)\right) .
$$

The spatial resources needed for the measurement process are one $(n+q)$-bit register to hold the digital representation of $|x\rangle$, and several $q$-bit registers that hold the $q^{2}$ measurement results (for each $\Sigma_{i}$ ) adding up to $p$. The measurement process we have constructed is thus efficient, belonging to the class P:P, i.e. polynomial in the input size and the output accuracy.

We point out that the preceding strategy cannot be used to efficiently evaluate $\|x\|^{2} \equiv\langle x|I| x\rangle$, because it requires $O(N)$ contributions to be added together. For that reason, to carry out efficient measurements, we need to restrict ourselves to measuring observables such that $\|x\|$ is either known (e.g. unitary evolution) or cancels out (e.g. ratios of expectation values).

\section{Efficient Solution of Linear Simultaneous Equations}

Now we turn to application of our measurement prescription to other linear algebra problems. Consider the problem of solving $A x=b$, where $A$ is an $N \times N$ matrix and $x, b$ are $N$-component vectors. The formal solution, $x=A^{-1} b$, involves matrix inversion. Exact computation of the matrix inverse is time consuming, e.g. Gaussian elimination requires $O\left(N^{3}\right)$ computational effort. So approximate iterative methods are frequently used to solve this linear algebra problem, especially when the matrix $A$ is sparse. They generate a sequence of approximate solutions, until a termination criterion signals that convergence up to a specified accuracy has been achieved. These methods generically work in the Krylov space, $\mathcal{K}_{r}\left(A, x_{0}\right)=\operatorname{span}\left\{x_{0}, A x_{0}, A^{2} x_{0}, \ldots, A^{r-1} x_{0}\right\}$, starting with an initial guess $x_{0}$. For a sparse $A$, each matrix-vector multiplication is $O(N)$, and this space can be covered with $O(r N)$ effort.

The problem is singular when an eigenvalue of the matrix $A$ approaches zero, and so the iterative convergence depends on the matrix condition number $\kappa$ that is the ratio of the largest to the smallest 
eigenvalue magnitudes. Within this context, the computational complexity of approximate iterative inversion methods is characterised in terms of the matrix size $N$, the desired solution accuracy $\epsilon$ and the matrix condition number $\kappa$. For the popular conjugate gradient algorithm [13], the computational complexity of solving $A x=b$ on classical computers is $O(d N \sqrt{\kappa} \log (1 / \epsilon))$, for a positive definite and $d$-sparse $A$.

In this Section, we present a simple quantum algorithm for computation of $x=A^{-1} b$ based on the Newton-Raphson method. We assume that the matrix $A$ has certain physically motivated properties, analogous to those of $H$ in the Hamiltonian evolution problem: (i) the Hilbert space is a tensor product of many small components, (ii) the components have only local interactions that make $A$ sparse, and (iii) both $A$ and $b$ are specified in terms of a finite number of efficiently computable functions, so that the resources needed to write them down do not influence the computational complexity. On the other hand, we do not need to assume that $A$ is positive definite. Then the algorithm is easy to implement with our digital representation of quantum states, and has computational complexity $O\left(d n \kappa^{2} \log ^{4}(1 / \epsilon)\right)$.

\subsection{Solution by Newton-Raphson Method}

The Newton-Raphson method can be used to iteratively solve an algebraic equation, when the derivatives of the functions involved can be easily obtained. It can be applied to the matrix inversion problem, with the number of significant digits approximately doubling with every iteration [14.

Let $B_{0}$ be a suitable initial guess for $A^{-1}$. Then, in terms of the residual matrix $R=I-B_{0} A$, the partial sum

$$
B_{r}=\left(1+R+R^{2}+\ldots+R^{r}\right) B_{0}
$$

converges to $A^{-1}$ as $n \rightarrow \infty$. The Newton-Raphson recurrence relation,

$$
B_{2 r+1}=2 B_{r}-B_{r} A B_{r}, \quad \mathrm{r}=0,1,3,7,15, \ldots,
$$

converges quadratically to $A^{-1}$, doubling the order $r$ at each stage. The iterative solution, $x_{r}=B_{r} b$, satisfies

$$
x_{r+1}=x_{r}+B_{0}\left(b-A x_{r}\right),
$$

and converges geometrically to the desired solution:

$$
\begin{aligned}
x_{r+1}-x & =\left(I-B_{0} A\right)\left(x_{r}-x\right), \\
& =\left(I-B_{0} A\right)^{r+1}\left(x_{0}-x\right), \\
& =-\left(I-B_{0} A\right)^{r+2} x .
\end{aligned}
$$

Formally, $\left\|x_{r}-x\right\| \leq\left\|I-B_{0} A\right\|^{r+1} \cdot\|x\|$ provides the fractional accuracy bound for $x$. We can always scale the problem such that $\|A\|$ is $\Theta(1)$. Then $\left\|A^{-1}\right\|=\Theta(\kappa)$, and $\|x\|=\Theta(\kappa\|b\|)$.

A good initial choice is $B_{0}=\alpha A^{\dagger}$, with $\alpha=2 /\left(\lambda_{\min }+\lambda_{\max }\right)$ in terms of the eigenvalues of $A^{\dagger} A$ [15]. The condition number of $A^{\dagger} A$ is $\lambda_{\max } / \lambda_{\min }=\kappa^{2}$. That makes

$$
\left\|I-B_{0} A\right\|=\left|\frac{\lambda_{\max }-\lambda_{\min }}{\lambda_{\max }+\lambda_{\min }}\right|=\frac{\kappa^{2}-1}{\kappa^{2}+1} .
$$

Each iteration of Eq. (33) requires two matrix-vector products. So the computational complexity of the algorithm, for reaching the accuracy $\|\Delta x\|<\epsilon\|x\|$, is

$$
2 r \mathcal{C}=O\left(\frac{2 \log (1 / \epsilon)}{\log \left(1 /\left\|I-B_{0} A\right\|\right)} \mathcal{C}\right)=O\left(\kappa^{2} \log (1 / \epsilon) \mathcal{C}\right),
$$

where $\mathcal{C}$ is the computational cost of a matrix-vector product. In practice, Frobenius bounds can be used to estimate the eigenvalue range of $A^{\dagger} A$, and the choice

$$
\alpha=\frac{1}{\sum_{j k}\left|A_{j k}\right|^{2}} \quad \text { or } \quad \alpha=\frac{1}{\left(\max _{j} \sum_{k}\left|A_{j k}\right|\right)\left(\max _{k} \sum_{j}\left|A_{j k}\right|\right)}
$$

guarantees convergence of the algorithm [15]. With our assumption that $A$ is specified in terms of a finite number of efficiently computable functions, such a choice of $\alpha$ can be easily made at the start of the algorithm. Note that this algorithm does not require the matrix $A$ to be positive definite. 
For sparse matrices, representing local interactions, calculation of the matrix-vector product can be efficiently parallelised and carried out in superposition on a quantum computer, as described in the next subsection. Coding the $N$ components of $x$ using $n=\log _{2} N$ qubits, we have $\mathcal{C}=O\left(d n q^{3}\right)$, where $d$ is the sparsity of the matrix and $q$ is the precision (number of register bits) required for individual arithmetic operations. As explained in Section 2, we can keep the round-off errors under control by choosing

$$
q=\Omega\left(\log \left(\frac{d r}{\epsilon\|x\|}\right)\right)=\Omega\left(\log \left(\frac{d r\|A\|}{\kappa \epsilon\|b\|}\right)\right) .
$$

The net computational complexity of the algorithm is, therefore,

$$
O\left(d n \kappa^{2} \log \left(\frac{1}{\epsilon}\right) \log ^{3}\left(\frac{d \kappa \log (1 / \epsilon)\|A\|}{\epsilon\|b\|}\right)\right) .
$$

While the (poly)logarithmic dependence of the computational complexity on the matrix size $N$ and the accuracy $\epsilon$ are desired features, the quadratic dependence on the condition number $\kappa$ remains a hurdle to be overcome. A known optimisation strategy is to use parallelisable preconditioners to effectively reduce $\kappa$ (see for example, Ref. [9]). The existence and construction of such preconditioners, however, depends on the detailed properties of the matrix to be inverted (e.g. FFT for translationally invariant matrices).

For comparison, the classical conjugate gradient algorithm also converges geometrically to the desired solution, but requires the matrix to be inverted to be positive definite in order to guarantee convergence. When $A$ is not positive definite, the problem solved in practice is $A^{\dagger} A x=A^{\dagger} b$, even at the cost of squaring the matrix condition number. Its computational complexity is then $O\left(\kappa \log (1 / \epsilon) \mathcal{C}_{C G}\right)$ when $A$ is not positive definite, and $O\left(\sqrt{\kappa} \log (1 / \epsilon) \mathcal{C}_{C G}\right)$ when $A$ is positive definite, where $\mathcal{C}_{C G}$ is the computational effort to implement a single iteration of the algorithm. $\mathcal{C}_{C G}$ involves matrix-vector multiplications, which can be easily parallelised for sparse matrices, and calculation of inner products for evolving the solution vector in the Krylov space along orthogonal directions, which cannot be parallelised. Overall, $\mathcal{C}_{C G}=O\left(d N q^{3}\right)$. Evolution along orthogonal directions makes the conjugate gradient algorithm converge faster than our Newton-Raphson method based algorithm, in terms of the dependence on $\kappa$. That is the price paid for bypassing the calculation of inner products, in order to achieve quantum superposition that reduces the computational complexity dependence from $N$ to $n$.

Quantum algorithms that reduce the computational complexity dependence from $N$ to $n$ have been proposed before; the algorithm of Ref. [8] has computational complexity $O\left(d^{2} n \frac{\kappa^{2}}{\epsilon}\right)$, and the algorithm of Ref. [9] improved the dependence on $\kappa$ using a preconditioning matrix. Both these algorithms are based on Hamiltonian simulation of $e^{i A t}$. The phase estimation technique is used to estimate the eigenvalues of $A$ up to a specified level of precision, and then the matrix is inverted in the spectral basis. Phase estimation has to evaluate the eigenvalues with error $O\left(\frac{\epsilon}{\kappa}\right)$, which contributes the dominant factor $\frac{\kappa}{\epsilon}$ to the computational complexity. Using a different strategy, we have achieved an exponential improvement in the dependence of the computational complexity on $\epsilon$.

\subsection{Digital State Implementation}

Evaluation of $x_{r}$ by iterating Eq.(33), starting with $x_{0}=\alpha A^{\dagger} b$, requires multiplication of a vector by a constant, addition of two vectors, and multiplication of a matrix with a vector. The first two operations are easily carried out with the digital representation described in Section 2 Multiplication of a matrix with a vector, on the other hand, has to be carefully implemented such that quantum superposition converts its computational complexity from classical $O(N)$ to quantum $O(n)$.

A simple way to parallelise multiplication of the sparse matrix with a vector is to decompose the matrix as a sum of block-diagonal parts, $A=\sum_{i=1}^{d} A_{i}$, with each part consisting of a large number of mutually independent blocks. Any sparse matrix can be efficiently decomposed in this manner, according to Vizing's theorem, using an edge-colouring algorithm for the corresponding graph. Then each colour represents a part, and each part contains $O(N / 2)$ mutually independent $2 \times 2$ blocks 6 Simultaneously carrying out individual block calculations for each part with a superposition of their block labels, and evaluating the contribution of each part in succession, the total computational effort for sparse matrix-vector multiplication becomes $O(d \log (N / 2))$ times the effort for a single $2 \times 2$ block multiplication.

\footnotetext{
${ }^{6}$ In many physical problems, the matrix $A$ is Hermitian and the off-diagonal elements, $A_{j, j+\mu}=A_{j+\mu, j}^{*}$, can be denoted by a single edge of the graph. Otherwise, the edges of the graph have to be directed.
} 


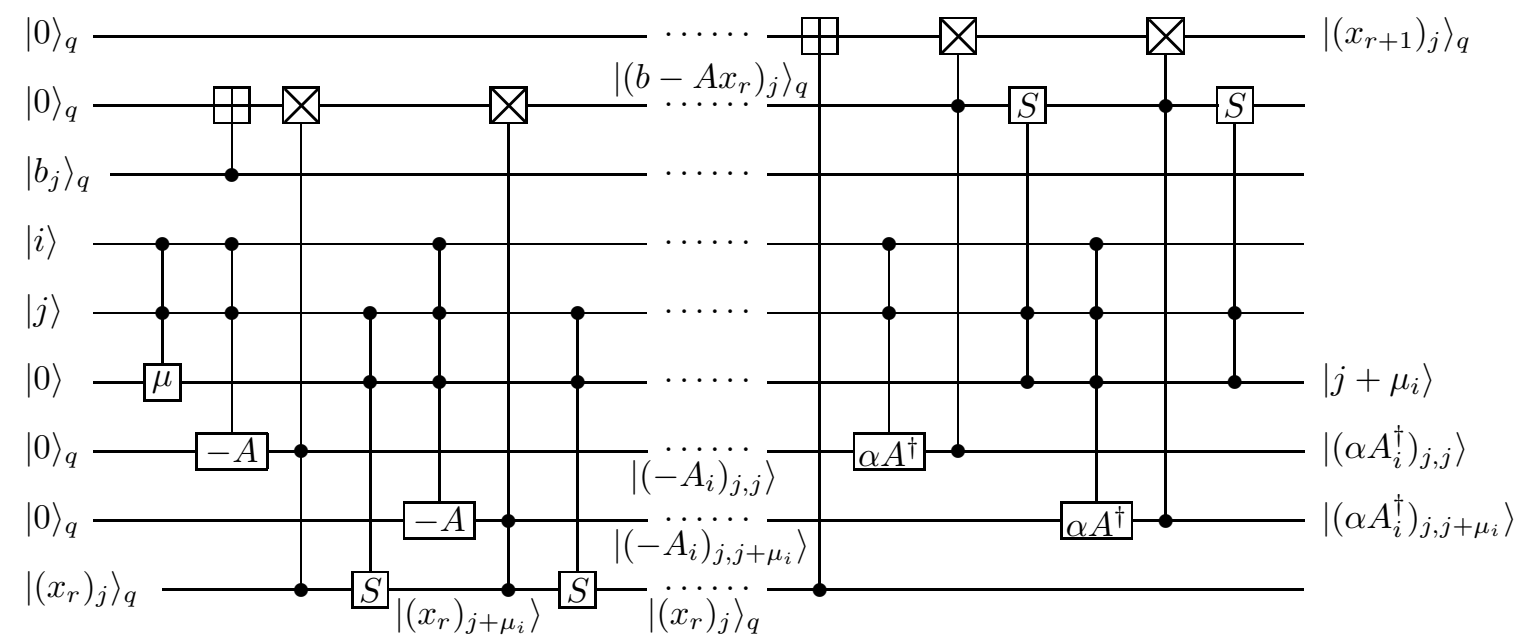

Figure 2: Digital quantum logic circuit for implementing the recursion relation, Eq.(33), to be executed with a uniform superposition over the index $j$. Operations for a single $A_{i}$ containing only $2 \times 2$ blocks (labeled by $\left.j, j+\mu_{i}\right)$ are shown. The two sparse matrix-vector multiplications involved in Eq.(33) are separated by "...", and a sum over the index $i$ is implicit in each of these two parts. Among the controlled logic gates, $\mu,-A$ and $\alpha A^{\dagger}$ denote oracle operations specified by the matrix, $S$ is the swap operation of Eq. (42), 区 stands for the generalised Toffoli gate implementing $|a, b, c\rangle \rightarrow|a, b, c+a b\rangle$, and $\boxplus$ labels generalised C-not gates performing $|a, b\rangle \rightarrow|a, b+a\rangle$.

In the digital representation, multiplication by diagonal matrix elements is straightforward, and multiplication by off-diagonal matrix elements of the $2 \times 2$ blocks becomes straightforward provided one can swap the $q$-bit register values, i.e.

$$
|j\rangle\left|x_{j}\right\rangle+|j+\mu\rangle\left|x_{j+\mu}\right\rangle \longrightarrow|j\rangle\left|x_{j+\mu}\right\rangle+|j+\mu\rangle\left|x_{j}\right\rangle .
$$

Such a swap operation is performed by the reflection operator,

$$
S=\sigma_{1} \otimes I^{\otimes q}, \quad S^{2}=I,
$$

acting on the subspace $\{|j\rangle,|j+\mu\rangle\} \otimes\left\{\left|x_{j}\right\rangle,\left|x_{j+\mu}\right\rangle\right\}$. The swap can be undone after the off-diagonal matrix element multiplication for a particular part $A_{i}$, to use $\left|x_{j}\right\rangle$ again for the next part.

The digital circuit implementation of Eq. (33), is schematically illustrated in Fig, 2 , For the sake of clarity, only the steps corresponding to a single $A_{i}$ are shown, with the sum over $i$ left implicit. The oracles have computational complexity $O\left(q^{3}\right)$ arising from evaluation of the matrix elements; the rest of the linear algebra operations have computational complexity $O\left(q^{2}\right)$. Including contributions of all the parts, and the computational effort needed to superpose the index $j$, we thus have the time complexity $\mathcal{C}=O\left(d n q^{3}\right)$ per iteration. We also note that the space resources required to put together the final solution $|x\rangle$ are a fixed number of $n$-bit and $q$-bit registers, because these registers can be reused.

We repeat that in our construction based on the digital representation for the quantum states, the full quantum advantage that reduces $N$ to $n$ in the computational complexity arises from a simple superposition of the quantum state label $j$, and this superposition in turn requires decomposition of the matrix into block-diagonal parts.

Once a sufficiently accurate solution for $|x\rangle=A^{-1}|b\rangle$ is obtained in a quantum register, the methodology of the Section 3 can be used to efficiently evaluate $k$-local observables $\langle x|O| x\rangle$.

\section{$5 \quad$ Efficient Matrix Exponentiation}

Efficient exponentiation of a Hermitian matrix $A$ is another algorithm that we construct, assuming that $A$ satisfies the three properties listed in the beginning of Section 4 . We restrict ourselves to evaluation of $e^{-A t}|b\rangle$, for given $t$ and $|b\rangle$, which is the combination often encountered in physical applications. 
The eigenvalue spectrum of any bounded Hermitian matrix is within a range $\left[\lambda_{\min }, \lambda_{\max }\right]$. With a linear transformation, this range can be mapped to any desired real interval, and the additive and the multiplicative constants can be handled by simple rescaling. For example,

$$
\tilde{A}=\left(A-\lambda_{\min } I\right) /\left(\lambda_{\max }-\lambda_{\min }\right),
$$

makes $\tilde{A}$ positive semidefinite, with eigenvalues in $[0,1]$. Then $e^{-A t}=e^{-\lambda_{\min } t} e^{-\tilde{A} \tilde{t}}$, with

$$
\tilde{t}=t\left(\lambda_{\max }-\lambda_{\min }\right) .
$$

In situations where $\lambda_{\min }$ and $\lambda_{\max }$ are not exactly known, respectively lower and upper bounds for them can be used. Frobenius bounds are convenient, based on the constraint that every eigenvalue of $A$ lies in one of the disks centred at $A_{j j}$ with radius $\sum_{l \neq j}\left|A_{l j}\right|$, and can be easily obtained for an $A$ that is specified in terms of a finite number of efficiently computable functions. Henceforth, we assume that such a rescaling of $A$ has been carried out as per the need of the algorithm, and drop the tilde's on $A$ and $t$ for simplicity.

\subsection{Chebyshev Expansion and its Complexity}

Chebyshev polynomials provide uniform approximations for bounded functions, with fast convergence of the series [18, 19. We scale $A$ such that its eigenvalue spectrum is within the domain $[-1,1]$ of the Chebyshev polynomials $T_{m}(x)=\cos \left(m \cos ^{-1} x\right)$. Then $\left\|e^{-A t}\right\|=\Theta\left(e^{t}\right)$, and

$$
e^{-A t}=\sum_{k=0}^{\infty} C_{k}(t) T_{k}(A)
$$

where the expansion coefficients are the Bessel functions:

$$
\begin{aligned}
C_{0} & =\frac{1}{\pi} \int_{0}^{\pi} e^{-t \cos \theta} d \theta=I_{0}(t), \\
C_{k>0} & =\frac{2}{\pi} \int_{0}^{\pi} e^{-t \cos \theta} \cos (k \theta) d \theta=2(-1)^{k} I_{k}(t) .
\end{aligned}
$$

Note that the Chebyshev polynomials are bounded in their domain, and the coefficients $I_{k}(t)=t^{k} /\left(2^{k} k !\right)+\ldots$ fall off faster by a factor of $2^{k}$ compared to the corresponding coefficients $t^{k} / k$ ! of the Taylor series expansion. This is the well-known advantage of the Chebyshev expansion compared to other series expansions.

The modified Bessel functions obey $I_{k}(t)>0$ for $t>0$, and increase with $t$ monotonically. Furthermore,

$$
I_{k}(t)=\sum_{s=0}^{\infty} \frac{(t / 2)^{k+2 s}}{s !(k+s) !}<\frac{(t / 2)^{k}}{k !} \exp \left(\frac{t^{2}}{4(k+1)}\right) .
$$

Therefore, when the Chebyshev expansion in Eq.(46) is truncated at order $r$, the truncation error is bounded by

$$
\begin{aligned}
\sum_{k=r+1}^{\infty} 2 I_{k}(t) & <\sum_{k=r+1}^{\infty} 2 \frac{(t / 2)^{k}}{k !} e^{t^{2} / 4(k+1)} \\
& <2 \frac{(t / 2)^{r+1}}{(r+1) !} e^{t^{2} / 4(r+2)}\left(1-\frac{t}{2(r+2)}\right)^{-1} .
\end{aligned}
$$

To control this truncation error, we choose

$$
r+2 \geq t
$$

and then demand

$$
\sum_{k=r+1}^{\infty} 2 I_{k}(t)<4 \frac{(t / 2)^{r+1}}{(r+1) !} e^{t^{2} / 4(r+2)}<\epsilon_{0} .
$$

The formal solution, consistent with Eq.(51), is

$$
r=\frac{e^{5 / 4} t}{2}+\ln \left(1 / \epsilon_{0}\right)=O\left(t+\log \left(1 / \epsilon_{0}\right)\right),
$$


and the fractional accuracy of this truncation is $\epsilon=e^{-t} \epsilon_{0}$.

A truncated series of the Chebyshev expansion is efficiently evaluated using Clenshaw's algorithm, based on the recursion relation

$$
T_{k+1}(A)=2 A T_{k}(A)-T_{k-1}(A),
$$

To evaluate $e^{-A t}|b\rangle$, one initialises the vectors $\left|y_{r+1}\right\rangle=0,\left|y_{r}\right\rangle=C_{r}|b\rangle$, and then uses the reverse recursion

$$
\left|y_{k}\right\rangle=C_{k}|b\rangle+2 A\left|y_{k+1}\right\rangle-\left|y_{k+2}\right\rangle,
$$

from $k=r-1$ to $k=0$. At the end,

$$
\sum_{k=1}^{r} C_{k} T_{k}(A)|b\rangle=\left(C_{0}|b\rangle+\left|y_{0}\right\rangle-\left|y_{2}\right\rangle\right) / 2
$$

is obtained using $r$ sparse matrix-vector products involving $A$. The computational complexity of the procedure is then

$$
O\left(r \mathcal{C}_{C}\right)=O\left(\left(t+\log \left(1 / \epsilon_{0}\right)\right) \mathcal{C}_{C}\right)
$$

where $\mathcal{C}_{C}$ is the computational cost of implementing the recursion of Eq.(155). As per the digital state implementation described in the next subsection, $\mathcal{C}_{C}=O\left(d n q^{3}\right)$.

The Bessel functions $I_{k}(t)$ up to order $r$ can be efficiently calculated to high precision, using the recursion relation

$$
I_{k-1}(t)=\frac{2 k}{t} I_{k}(t)+I_{k+1}(t),
$$

in descending order [20]. One starts with approximate guesses for $I_{l}(t)$ and $I_{l+1}(t)$, with $l$ slightly larger than $r$, and uses the recursion relation repeatedly to reach $I_{0}(t)$. Then all the values are scaled to the correct normalisation by imposing the constraint $I_{0}(t)+2 \sum_{k=1}^{[l / 2\rceil} I_{2 k}(t)=e^{t}$. This procedure to determine the expansion coefficients requires $\Theta\left(r q^{2}\right)$ computational effort, and so does not alter the overall computational complexity given by Eq.(57).

To control the round-off error while summing up the Chebyshev expansion, the coefficients $C_{k}$ up to order $r$, and the elements of matrix $A$, have to be evaluated to $q=\Omega\left(\log \left(\frac{d r}{\epsilon}\right)\right)$ bit precision, as explained in Section $2^{7}$ The net computational complexity of the algorithm is, therefore,

$$
O\left(d n\left(t+\log \left(\frac{1}{e^{t} \epsilon}\right)\right) \log ^{3}\left(\frac{d\left(t+\log \left(1 / e^{t} \epsilon\right)\right)}{\epsilon}\right)\right) .
$$

The desirable features of this computational complexity are the linear dependence on $n$ and $t$, and the (poly)logarithmic dependence on $\epsilon$. This behaviour is similar to the scaling in Eq.(18) for the Hamiltonian evolution problem.

\subsection{Digital State Implementation}

Summation of the series in Eq.(46), truncated to order $r$, requires $r$ executions of the Clenshaw recursion relation, Eq.(55). For a sparse Hermitian $A$, that can be implemented in the same manner as described in Section 4.2. The digital circuit implementation of Eq.(55), for a single part $A_{i}$, is schematically illustrated in Fig 3. It has computational complexity $O\left(q^{3}\right)$ arising from evaluation of the matrix elements; the rest of the linear algebra operations contribute computational complexity $O\left(q^{2}\right)$. Including contributions of all the parts, and the computational effort needed to superpose the index $j$, we thus have the time complexity $\mathcal{C}_{C}=O\left(d n q^{3}\right)$. We also note that the space resources required to put together the full Chebyshev expansion are a fixed number of $n$-bit registers and $O(r) q$-bit registers.

\subsection{Reduction of Matrix Inverse to Matrix Exponentiation}

For a positive definite matrix, the matrix inverse problem with accuracy $\epsilon$ can be reduced to the matrix exponentiation problem, using the result [16]

$$
(1-\epsilon) a^{-1} \leq \sum_{j=p}^{p^{\prime}} h e^{j h} e^{-a e^{j h}} \leq(1+\epsilon) a^{-1},
$$

\footnotetext{
${ }^{7}$ Note that $q$ is determined by the fractional accuracy of the intended result.
} 


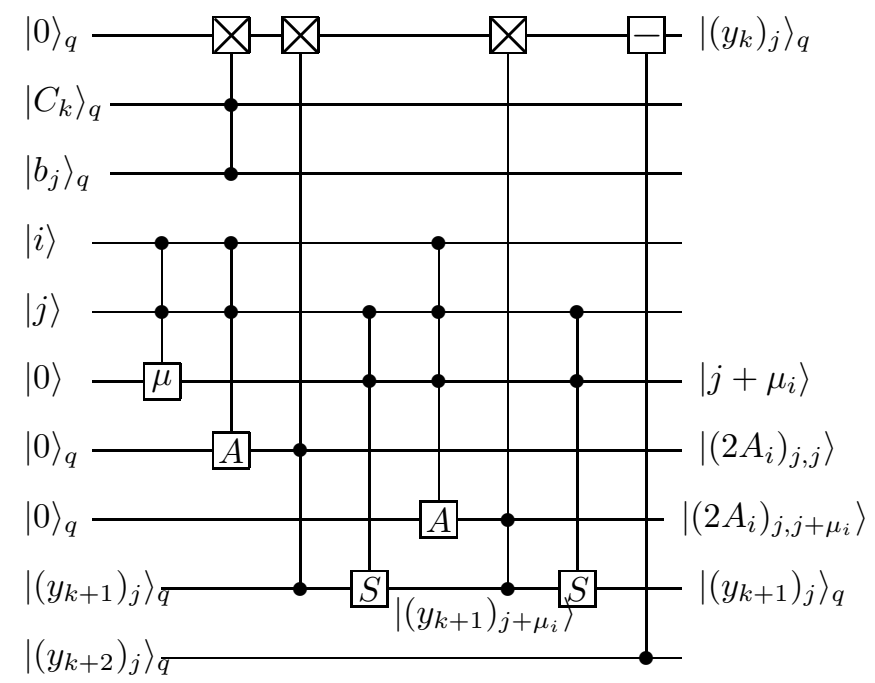

Figure 3: Digital quantum logic circuit for executing the recursion relation of Clenshaw's algorithm, Eq.(55), to be executed with a uniform superposition over the index $j$. Operations for a single $A_{i}$ containing only $2 \times 2$ blocks (labeled by $j, j+\mu_{i}$ ) are shown. Among the controlled logic gates, $\mu$ and $A$ denote oracle operations specified by the matrix, $S$ is the swap operation of Eq.(42), $₫$ stands for the generalised Toffoli gate implementing $|a, b, c\rangle \rightarrow|a, b, c+a b\rangle$, and $\boxminus$ labels the generalised C-not gate performing $|a, b\rangle \rightarrow|a, b-a\rangle$.

for all $a \in\left[\frac{1}{\kappa}, 1\right]$. Here $\epsilon$ is chosen as the fractional accuracy for $a^{-1}$, since $a^{-1}$ is singular when $\kappa \rightarrow \infty$. This result follows from approximating the integral

$$
a^{-1}=\int_{0}^{\infty} e^{-a t} d t \stackrel{t=e^{y}}{=} \int_{-\infty}^{\infty} e^{y-a e^{y}} d y
$$

by the trapezoidal rule, and bounding the error using the Euler-Maclaurin formula. The choice of the discretisation parameters as [16]

$$
\begin{gathered}
h=\frac{2 \pi}{e^{2}(2 n+1)^{2}}, \quad n=\left\lceil\frac{1}{2} \ln \frac{24}{\epsilon}\right\rceil, \\
p=\left\lfloor-\frac{1}{h} \ln \frac{3}{\epsilon}\right\rfloor, \quad p^{\prime}=\left\lceil\frac{1}{h} \ln \left(\kappa \ln \frac{3}{\epsilon}\right)\right\rceil,
\end{gathered}
$$

makes the computational effort needed for the reduction in Eq. that for the variable $t=e^{j h}$, we have

$$
j \in\left[p, p^{\prime}\right] \Longrightarrow t \in[\epsilon / 3, \kappa \ln (3 / \epsilon)] .
$$

The result of Eq. (60) can be applied to positive definite matrices, with $a \rightarrow A$, interpreting all matrix functions as their power series expansions. Then rapidly converging Chebyshev expansion for $\exp (-A t)$ can be used, as described in Section 5.1 for every value of $j$.

The trapezoidal rule sum of Eq.(60) has $p^{\prime}-p+1$ terms, and its error has to be bounded by $\epsilon_{0} \leq \kappa \epsilon$. That can be achieved by choosing the Chebyshev expansion truncation order, as per Eq.(53),

$$
r_{j}=O\left(t+\log \left(\frac{p^{\prime}-p}{\epsilon_{0}}\right)\right)=O\left(e^{j h}+\log \left(\frac{\log ((\kappa / \epsilon) \log (1 / \epsilon))}{\kappa \epsilon}\right)\right) .
$$

The total number of times the recursion relation of Eq.(55) needs to be executed is then

$$
\begin{aligned}
r_{\mathrm{tot}}=\sum_{j=p}^{p^{\prime}} r_{j} & =O\left(\frac{\kappa}{h} \log \frac{1}{\epsilon}+\frac{1}{h} \log \left(\frac{\kappa}{\epsilon} \log \frac{1}{\epsilon}\right) \log \left(\frac{\log ((\kappa / \epsilon) \log (1 / \epsilon))}{\kappa \epsilon}\right)\right) \\
& =O\left(\kappa \log ^{3}(1 / \epsilon)+\log ^{4}(1 / \epsilon)\right) .
\end{aligned}
$$


For any fixed $b$, the fractional error in evaluating $x=A^{-1} b$ is the same as the fractional error in evaluating $A^{-1}$. The preceding algorithm has two sources of error: replacement of the integral by the Euler-Maclaurin formula and truncation of the Chebyshev expansion. Putting all the pieces together, the computational complexity of this algorithm for solving the set of linear equations with accuracy $\|\Delta x\|<2 \epsilon\|x\|$, is $O\left(r_{\text {tot }} \mathcal{C}_{C}\right)$. Also, the required digital precision $q$ is the same as in Eq.(40), with $r$ replaced by $r_{\text {tot }}$. Compared to Eq.(38), we see that the dependence of the computational complexity on $\log (1 / \epsilon)$ is worse, although the dependence on $\kappa$ is better when $A$ is positive definite. Overall, the simplicity of implementation clearly favours the algorithm of Section 4 for solution of linear simultaneous equations.

\subsection{Potential Applications}

With our digital representation, we can easily construct useful algorithms involving unnormalised quantum states. Consider determination of expectation values of various physical quantities in the ground state of a quantum system. For Hamiltonian systems with a spectral gap $\Delta$, a convenient way to obtain the ground state $\left|\psi_{0}\right\rangle$ is to evolve an approximate ansatz for the ground state $|\psi\rangle$ in Euclidean time:

$$
e^{-H T}|\psi\rangle=e^{-E_{0} T}\left|\psi_{0}\right\rangle\left(1+O\left(e^{-\Delta T}\right)\right) .
$$

The l.h.s. can be efficiently calculated by the methods presented earlier in this Section, and then ratios of ground state expectation values can be obtained even when the ground state energy $E_{0}$ is not known.

Going further, problems in statistical mechanics frequently involve systems in equilibrium with a heat bath. Such a system with the Hamiltonian $H$ is described by the thermal state

$$
\rho=e^{-\beta H} / Z, Z=\operatorname{Tr}\left(e^{-\beta H}\right) .
$$

Physically observable quantities are then obtained as the expectation values

$$
\left\langle O_{a}\right\rangle=\operatorname{Tr}\left(\rho O_{a}\right) .
$$

For bounded Hamiltonians, $e^{-\beta H}$ is completely well-behaved, and an expansion of $\rho$ in powers of $\beta$ has a non-zero radius of convergence around $\beta=0$ (i.e. infinite temperature). Singular critical phenomena arise from the large degeneracy of states that contribute to the partition function $Z$. Our techniques, described earlier in this Section, allow efficient calculation of $e^{-\beta H}$ acting on a vector. So by decomposing $O_{a}$ in the Pauli operator basis (as described in Section 3), we can efficiently evaluate ratios of thermal state expectation values (where $Z$ cancels out).

\section{Summary and Outlook}

The quantum Hilbert space allows superposition of $N=2^{n}$ independent components using $n$ qubits. They can then all be simultaneously processed in the single-instruction-multiple-data mode, familiar from the design of parallelisable algorithms for classical computers. This is a key ingredient in development of quantum algorithms that can be exponentially faster than their classical counterparts. Such a conversion of parallelisation into superposition is not possible for generic computational problems, but it can be achieved for many linear algebra problems, by domain decomposition of the algorithms and breakdown of matrix operations into block-diagonal ones.

On the other hand, this exponential advantage of quantum superposition is severely limited by the fact that only $n$ bit worth of information can be extracted from the result at the end. So the overall algorithm is efficient only when the final observables are local in some sense. Although no general prescription is available, we have shown that $k$-local observables appearing in evaluations of $k$-point Green's functions of quantum many body problems can be efficiently evaluated.

An important component of our demonstration is the digital representation of quantum states [5]. It makes linear algebra calculations straightforward to perform. But, much more importantly, it also allows a bit-by-bit deterministic evaluation of the expectation values, instead of a probabilistic one. The well-known Chernoff bound strategy can then be used to make the measurement effort logarithmic with respect to the output accuracy. Thus, parallelisable algorithms with $k$-local observables become efficient with respect to both the input and output sizes, and the problems they solve belong to the computational complexity class that we have labeled P:P. 
Our quantum measurement strategy can be applied to any output state of a quantum algorithm, as in the Hamiltonian evolution problem. But a useful feature of the digital representation is that it is not constrained by unitary evolution at every step, and so one can easily incorporate in it non-unitary evolution steps such as series expansions. We have used this flexibility to construct efficient quantum algorithms for two non-unitary but practical problems: solution of simultaneous linear equations using the Newton-Raphson method, and exponentiation of a matrix using the Chebyshev expansion. It would certainly be worthwhile to explore other problems that can be solved efficiently using our methods.

Our algorithm construction is explicit, and not reductionist, which clearly demonstrates how the algorithms work in practice. It also illustrates how the decomposition of matrices into block-diagonal components and the digital representation of quantum states are closely tied, respectively, to the input and the output efficiency of our algorithms. The classical ingredients used in our algorithms - parallelisation of linear algebra operations, digital representation, Chernoff bound, Newton-Raphson method, Chebyshev expansion - are all well-known. Our contribution has been to put them together in a manner that successfully carries over their advantages into the quantum domain.

\section{References}

[1] R.P. Feynman, Simulating physics with computers, Int. J. Theor. Phys. 21 (1982) 467-488.

[2] R.P. Brent and P. Zimmermann, Modern Computer Arithmetic, (Cambridge University Press, 2010).

[3] D.W. Berry, A.M. Childs, R. Cleve, R. Kothari and R.D. Somma, Exponential improvement in precision for simulating sparse Hamiltonians, in Proc. 46th Annual ACM Symp. on Theory of Computing, STOC'14, New York, NY, 31 May-3 June (ACM, New York, 2014), pp.283-292.

[4] D.W. Berry, A.M. Childs, R. Cleve, R. Kothari and R.D. Somma, Simulating Hamiltonian dynamics with a truncated Taylor series, Phys. Rev. Lett. 114 (2015) 090502.

[5] A. Patel and A. Priyadarsini, Optimisation of Quantum Hamiltonian Evolution: From Two Projection Operators to Local Hamiltonians, Int. J. Quantum Inform. 16 (2016) $165002 \%$.

[6] S. Lloyd, Universal quantum simulators, Science 273 (1996) 1073-1078.

[7] D. Aharonov and A. Ta-Shma, Adiabatic quantum state generation and statistical zero knowledge, in Proc. 35th Annual ACM Symp. on Theory of Computing, STOC'03, San Diego, CA, 9-11 June (ACM, New York, 2003), pp.20-29.

[8] A.W. Harrow, A. Hassidim and S. Lloyd, Quantum algorithm for solving linear systems of equations, Phys. Rev. Lett. 103 (2009) 150502.

[9] B.D. Clader, B.C. Jacobs and C.R. Sprouse, Preconditioned quantum linear system algorithm, Phys. Rev. Lett. 110 (2013) 250504.

[10] T. Cai, D. Kim, Y. Wang, M. Yuan and H.H. Zhou, Optimal large-scale quantum state tomography with Pauli measurements, Ann. Statistics 44 (2016) 682-712.

[11] M.A. Nielsen and I.L. Chuang, Quantum Computation and Quantum Information, (Cambridge University Press, Cambridge, 2000), Section 6.2.

[12] H. Chernoff, A measure of asymptotic efficiency for tests of a hypothesis based on the sum of observations, Ann. Math. Statistics 23 (1952) 493-507.

[13] M.R. Hestenes and E. Stiefel, Methods of conjugate gradients for solving linear systems, J. Res. National Bureau of Standards 49 (1952) 409-436.

[14] W.H. Press, S.A. Teukolsky, W.T. Vetterling and B.P. Flannery, Numerical Recipes: The Art of Scientific Computing, Third Edition, (Cambridge University Press, 2007), Chapter 2.5.

[15] V. Pan and J. Reif, Efficient Parallel Solution of Linear Systems, in Proc. 17th Annual ACM Symp. on Theory of Computing, STOC'85, Providence, RI, 6-8 May (ACM, New York, 1985), pp.143-152.

[16] S. Sachdeva and N.K. Vishnoi, Matrix inversion is as easy as exponentiation, arXiv:1305.0526. 
[17] S. Sachdeva and N.K. Vishnoi, Faster algorithms via approximation theory, FnT Theoretical Computer Science 9 (2013) 125-210.

[18] G.B. Arfken, H.J. Weber and F.E. Harris, Mathematical Methods for Physicists: A Comprehensive Guide, Seventh Edition, (Academic Press, 2011), Chapter 18.4.

[19] H. Tal-Ezer and R. Kosloff, An accurate and efficient scheme for propagating the time dependent Schrödinger equation, J. Chem. Phys. 81 (1984) 3967-3971.

[20] M. Abramowitz and I.A. Stegun (Eds.), Handbook of Mathematical Functions: With Formulas, Graphs and Mathematical Tables, (Dover Publications, 1965), Chapter 9.12. 